\title{
Further Specification Analysis of the Unique Path Constraint Effect: From the Perspective of Spatial Extension
}

\author{
Masaki Yasuhara ${ }^{1}$ \\ ${ }^{1}$ University of Tsukuba, Tennoudai 1-1-1, Tsukuba, Ibaraki, 305-8577, Japan \\ Correspondence: Masaki Yasuhara, University of Tsukuba, Tennoudai 1-1-1, Tsukuba, Ibaraki, 305-8577, Japan. \\ E-mail: myasuhara1986@yahoo.co.jp
}

Received: November 27, 2013

Accepted: December 17, 2013

Online Published: December 18, 2013

doi:10.5430/elr.v2n2p141

URL: http://dx.doi.org/10.5430/elr.v2n2p141

\begin{abstract}
It has been argued that a single clause may not include a change of state expression and a change of location expression (e.g., *Sam kicked Bill black and blue out of the room.). This co-occurrence restriction is known as the Unique Path Constraint effect. The purpose of this paper is to show that this constraint comes from the nature of event structure - assuming that an event structure can be composed of at most two internal events, a single event may not include more than one distinct change. I argue that apparent counterexamples (e.g., John broke the egg into the bowl.), which are presented in the literature, can also be explained under the event-based analysis provided in this paper. The co-occurrence of a change of state expression and a change of location expression in a single clause is possible when they are in a further specification relation. In such a case, the two expressions refer to different aspects of a single unified change, so they can be licitly associated with a single subevent. In this paper, I show that a further specification relation is satisfied when an event that is expressed by a change of state expression involves spatial extension, and the spatial extension is specified by a change of location expression. This paper provides empirical evidence for the event-based analysis of the Unique Path Constraint effect, and consequently provides empirical support for the idea that human language is devised assensitiveto event structure.
\end{abstract}

Keywords: Unique Path Constraint, Event Structure, Further Specification, Spatial Extension, Anchored Motion

\section{Introduction}

\subsection{The Unique Path Constraint}

It has been argued that a single clause may not involve both a change of state expression and a change of location expression (Simpson, 1983; Goldberg, 1991, 1995: Levin \& Rappaport, 1995; Tortora, 1998; Matsumoto, 2006; among others.). This restriction is known as the Unique Path Constraint (henceforth, the UPC) formulated in the following way (Goldberg, 1991:368):

Unique Path Constraint: if an argument $X$ refers to a physical object, then more than one distinct path cannot be predicated of $\mathrm{X}$ within a single clause.

The UPC prohibits the co-occurrence of multiple expressions that denote distinct paths in a single clause. Goldberg argues that a result phrase and a path phrase may not co-occur in a single clause in (3) because their co-occurrence violates the UPC.
(2) a. Sam kicked Bill black and blue.
b. Sam kicked Bill out of the room.
(3) a. * Sam kicked Bill black and blue out of the room.
b. * Sam kicked Bill out of the room black and blue.

(Goldberg, 1991:368)

The result phrase black and blue in sentence (2a) exhibits a change of state of the referent denoted by the object NP Bill. Likewise, the path phrase out of the room in sentence (2b) expresses a change of the location of Bill. Goldberg argues that the result and path phrases exhibit two distinct paths; the co-occurrence of the result phrase and the path phrase in a single clause violates the UPC, as in (3).

This co-occurrence restriction has been formulated in various ways in the literature (e.g., Single Delimiting 
Constraint (Tenny, 1994), Further Specification Constraint (Tortora, 1998), and Single Development Constraint (Matsumoto, 2006)). All these analyses share the insight that a single clause may express only one event or one change, in parallel fashion with the UPC.

Rappaport\& Levin (1998) suggest that the UPC effect follows from the nature of the event structure. In their event structure system, two distinct changes of state and of location are prohibited from co-occurring in the single result event, accounting for the infelicity of (3).

\subsection{Apparent Counterexamples}

This event structural account, however, appears to face empirical problems. The following examples, which are noted by many researchers (Goldberg, 1995, 2001; Levin \& Rappaport, 1995; Filip, 2003; Matsumoto, 2006; Okuno, 2003), appear to violate the UPC:

(4) a. The butcher sliced the salami onto the wax paper.

b. Joey grated the cheese onto a serving plate.

c. Sam shredded the papers into the garbage pail.

(Goldberg, 1995:171)

(5) a. The cook cracked the eggs into the glass.

b. $\quad$ Daphne shelled the peas onto the plate.

(Levin \& Rappaport, 1995:60)

Kelly broke the branch off the tree.

(Rappaport \& Levin, 1998:123)

All these sentences include matrix verbs denoting a change of state of the objects, which can be considered paths in terms of a change of state. Furthermore, they contain path phrases, displaying paths in terms of literal motion. Consequently, two distinct paths co-occur in a single clause in these sentences. In this way, these sentences appear to violate the UPC, and they could cast doubt on the validity of the event structural account.

Several previous studies have attempted to resolve these empirical problems(pragmatic conditions (Goldberg, 1995, 2001), polysemy-based approach (Levin \& Rappaport, 1995) and the Single Development Constraint account (Matsumoto, 2006)). These approaches, however, prove inadequate, as Yasuhara $(2012,2013)$ argues.

\subsection{Purpose}

In this paper, I show that the event-basedanalysis conducted inRappaport \& Levin (1998), which suggests that the UPC effect follows from the nature of event structure, is on the right track. I argue that the examples in (4)-(6), which are supposed to be counterexamples to the UPC by many researchers, are apparent counterexamples. Although these sentences appear to contain two distinct paths, I argue that one path further specifies the other; that is, the former specifies the underspecified part of the meaning of the latter. Specifically, I argue that path phrases can further specify a change of state denoted by verbs when the path phrases refer to the motion of only part of an object, which I call anchored motion. As a result, the two paths in these sentences constitute a unique path, and no UPC violation arises. This analysis empirically supports the general idea that the mechanism of event structure underlies grammatical phenomena (e.g., Levin \& Rappaport, 1995; Pinker, 1989; Pustejovsky, 1991;Ramchand, 2008; Randall, 2010;Rappaport \& Levin, 1998, 2001; Ritter \& Rosen, 1998; Yasuhara, 2012, 2013).

The organisation of this paper is as follows. First, section 2 presents an event structure analysis of the UPC effect. Section 3 shows that path phrases that denote a motion in which only part of an object moves along a path may further specify the change of state exhibited by verbs. Based on this observation, section 4 argues that the path phrases in (4)-(6) further specify the change of state denoted by the verbs. As a result, the change of state and change of location in these sentences form a unique change of state, and no UPC violation arises. Section 5 provides a further consideration of the Unique Path Constraint effect. Finally, section 6 presents concluding remarks.

\section{An Event-Based Analysis of the Unique Path Constraint Effect}

In this section, I argue that the co-occurrence restriction on a change of state expression and a change of location expression follows from the event structural restriction that prohibits a single event from involving two distinct results. Based on the event structural account of the UPC mentioned in section 1, I argue that a change of state denoted by the verbs and motion expressed by the path phrases in (4)-(6) constitute a single unified result in which the path phrases further specifythe change of state of the verbs. This analysis is in accordance with Goldberg's (1991) observation that the UPC does not rule out a co-occurrence of a change of state expression and a change of location expression in a single clause when the latter further specifies the former. 
The following discussions introduce some basic components of the theoretical framework proposed by Levin \&Rappaport (1995), Rappaport \& Levin $(1998,2001)$ and Tortora (1998). This theory gives an event structural account of the UPC effect.

On the basis of the aspectual distinctions proposed by Vendler (1957), Levin \& Rappaport (1995) develop several decompositional predicate representations. Events with accomplishment aspect, for example, are assumed to be composed of two events:

Pat broke the window. (Levin and Rappaport (1995:79))

[[ Pat DO-SOMETHING ] CAUSE [ the window BECOME BROKEN ]]

(Levin \& Rappaport, 1995:83, with modifications)

The sentence in (7) describes a situation in which Pat did something and as a result the window broke. Since Pat's doing something causes the window to break, his action can be regarded as a cause. The window's change of state, on the other hand, can be considered as a result caused by Pat's action. This causal relation can be captured by the decompositional predicate representation in (8). In this representation, the causing event is expressed by [ Pat DO-SOMETHING ] and the result event is designated by [ the window BECOME BROKEN ]. The causing event is connected with the result event by the function CAUSE, which represents a causal relation between them; the former causes the latter.

In this paper, I express a causal relation between a causing event and a result event in the following simpler way for convenience:

$$
\text { [[CAuse ] CAUSE [Result ]] }
$$

[[CAuse Pat did something ] CAUSE [Result the window broke ]]

The template in (9) is the basic representation of a causal relation between a causing event and a result event, in which [CAuse] denotes the causing event and [Result] the result event. In our representation, we assign the decompositional predicate representation in (10), rather than that in (8), to the sentence in (7).

Rappaport \& Levin (2001:791) assume that a single clause can include two events at most, where the two events are dependent on each other in terms of a causal relation. The template in (9), thus, is assumed to be the most complex structure. This means that any decompositional predicate representation of complex event structure has to be composed of a causing event and a result event, but it can neither be composed of two result events nor two causing events.

For this reason, when multiple expressions that denote result state or result location co-occur in a single clause, they must refer to a single result state or result location, but not distinct ones. Tortora (1998) calls this constraint the Further Specification Constraint, as exemplified by the following sentence:

The bottle broke open.

(Levin \& Rappaport, 1995:59)

This sentence involves the change of state verb break and the result phrase open. At first glance, this sentence appears to include two result events: the event in which the bottle broke, and that in which it became open. A close scrutiny of this sentence, however, reveals that the result phrase open further specifies change of state denoted by the verb break. (Note 1) This sentence can be considered to include a single unified result state, and therefore this sentence does not involve two distinct result events. The following decompositional predicate representation can be assigned to this sentence:

$$
\text { [[CAUSE ] CAUSE [RESult the bottle broke open ]] }
$$

In this representation, the change of state denoted by the verb break and that expressed by the result phrase open are both involved in the same result event: they refer to a single unified result state.

Given the theoretical framework presented here, we can give an event structural account of the sentences in (3), repeated here as (13):

(13) a. * Sam kicked Bill black and blue out of the room.

b. * Sam kicked Bill out of the room black and blue.

The sentences in (13) include the result phrase black and blue and the path phrase out of the room, which refer to a changes of state and of location of the participant denoted by the NP Bill, respectively. (Note 2) In these sentences, in contrast to (11), further specification does not hold between the result phrase and the path phrase. In other words, these two phrases refer to two distinct result events. Therefore, the co-occurrence of the result phrase and the path 
phrase is prohibited.

Thus far, we have discussed that the UPC follows from the nature of event structure. The examples in (4)-(6), repeated here as (14)-(16), however, appear to pose a serious problem for this account:

(14) a. The butcher sliced the salami onto the wax paper.

b. Joey grated the cheese onto a serving plate.

c. Sam shredded the papers into the garbage pail.

(15) a. The cook cracked the eggs into the glass.

b. Daphne shelled the peas onto the plate.

Kelly broke the branch off the tree.

These sentences include a change of state verb and a path phrase in a single clause and apparently contain two distinct paths in terms of change of state and change of location.

If the event structural account of the UPC effect presented here is on the right track, a change of state denoted by the verbs and a change of location expressed by the path phrases in these sentences should be involved in the same result events; that is, the path phrases should further specify the meaning of the verbs, in the same way as open in (11).

In the next section, we observe that a certain type of path phrases may further specify the meaning of change of state verbs. Based on this observation, I argue that the path phrases included in these examples serve to further specify the meaning of the verbs.

\section{Motion Involved in Change of Spatial Extent}

In section 3.1, I survey Goldberg (1991) and Lindner (1982, 1983), who discuss the co-occurrence of a change of state expression and a change of location expression in terms of further specification. Their analyses are mainly based on sentences denoting change of physical shape and position. In section 3.2, I propose that their analyses are applicable to change of state in terms of physical separation and combination. The argument presented in this section allows us to claim that the path phrases in (14)-(16) serve to further specify the meaning of the change of state verbs.

\subsection{The Unique Path Constraint and the Anchored Motion}

Goldberg (1991) provides an extensive study of the co-occurrence restriction on a change of state expression and a change of location expression in a single clause and formulates the UPC, as in (1), repeated here as (17):

Unique Path Constraint: if an argument $X$ refers to a physical object, then more than one distinct path cannot be predicated of $\mathrm{X}$ within a single clause.

At first sight, the UPC appears to ban any co-occurrence of a change of state expression and a change of location expression in a single clause, as in (18):
(18) a. * Sam kicked Bill black and blue out of the room.
b. * Sam kicked Bill out of the room black and blue.

In these sentences, a result phrase and a path phrase are prohibited from co-occurring in a single clause.

The UPC, however, does not always work this way. Witness the following data:

(19) a. John stood up straight.

b. He got down into a squatting position.

(Goldberg, 1991:373, with slight modifications)

In these sentences, the result phrases straight and into a squatting position co-occur with the path phrases up and down in a single clause, respectively. Here, the path phrases and the result phrases in these sentences do not refer to two distinct paths. For example, in (19a), the path phrase up specifies a motion that accompanies a change of position (becoming straight). The path phrase up and the result phrase straight refer to the unique path in terms of the change of position. In (19b), likewise, the change of position denoted by the result phrase into a squatting position is accompanied by the motion of a part of the body, which is referred to by the path phrase down. Thus, the examples in (19) include a unique path in terms of change of position and, therefore, do not violate the UPC. The situations described in (19a) and (19b) can be illustrated by the figures in (20a) and (20b), respectively: 
(20) a.

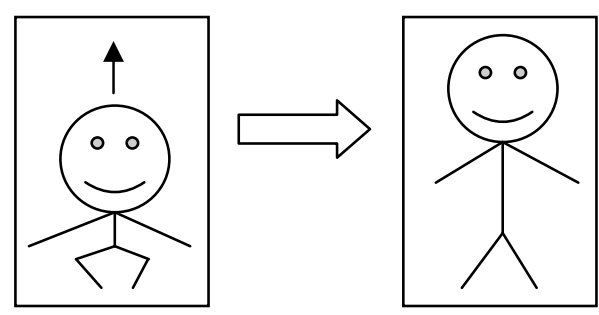

b.

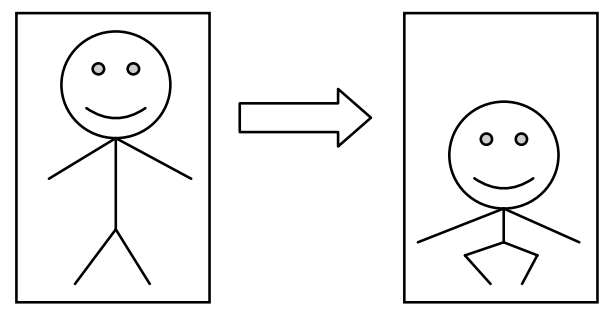

The rightward arrows show transitions of situations, where a situation in the left square changes into one in the right square. The left square of (20a) illustrates that John in (19a) is trying to stand up. As a result, he is standing straight in the right square of (20a). In (20b), similarly, the left square shows that the referent of he in (19b) is trying to get down, and consequently he is in a squatting position, as depicted in the right square. The upward and downward arrows in these figures represent directions of movements. Note that in both of the cases in (19a) and (19b), the legs are anchored at a fixed location (say, the ground), and what moves is only the upper half of the body. In this way, the change of physical position involves the motion of only part of an object.

A crucial difference between the sentences in (18) and (19) is whether a path phrase refers to the motion of only a part of an object. The path phrases in (18) express translational motion (Talmy (2000:25)), a movement that changes the location of an object. In (18), Bill is made to move from the inside of the room to the outside. The path phrase out of the room expresses a motion in which Bill changes his location. In (19), in contrast, the movements described by the path phrases up and down do not change the location of the referents of John and he. Hereafter, let us call this type of motion anchored motion. Anchored motion is defined as follows: (Note 3)

Anchored motion is the movement in which an object undergoing change remains anchored at a fixed location while rearranging parts of its extension in space.

Given the contrast in acceptability between (18) and (19), we can argue that a path phrase can co-occur with a change of state expression in a single clause when the path phrase refers to anchored motion; in this case, no UPC violation arises.

Similar observations are made in Lindner $(1982,1983)$. Lindner observes that verbs displaying spatial extension (i.e., a process in which an area covered by an object or a group of objects increases) such asspread and lengthen involve paths that are inherent in the spatial extension, and these paths can be referred to by out:
(22) a.
Stretch out the rope.
b.
Lengthen out your stride.

(Lindner, 1983:94)

In these sentences, the processes of stretching a rope and lengthening one's stride include motion that accompanies spatial extension, and the motion can be expressed by out.

Spatial reduction (i.e., a process in which an area covered by an object or a group of objects decreases) also involves motion, and the motion can be referred to by down (Lindner, 1982:321):

She \{scrunched / crunched \} down in the corner so no one would see her.

The action of scrunching or crunching involves spatial reduction, in whichthe space occupied by the referent denoted by the subject she decreases in height. The particle down denotes motion that accompanies this spatial reduction. In both cases in (22) and (23), the particles out and down refer to an anchored motion but not to a translational motion of objects undergoing a change of spatial extent (i.e., spatial reduction or extension).

To summarise the analyses of Goldberg (1991) and Lindner (1982,1983), a change of physical position and spatial extent inherently includes anchored motion, which can be referred to by path phrases. Spatial extension and 
reduction can be schematically represented by the following figures: (Note 4)

(24) a. Spatial extension

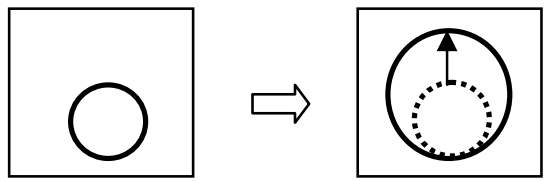

b.

Spatial reduction
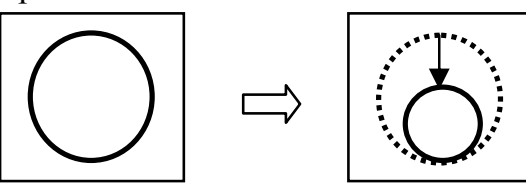

The upward and downward arrows in the squares represent motion that accompanies the change of spatial extent, and the rightward arrows show the transitions of situations. The figures in (24a) illustrate spatial extension, corresponding to the examples in (19a) and (22); an action of standing up and spatial extension involve motion from inward to outward. The figures in (24b), in contrast, show spatial reduction, which corresponds to the sentences in (19b) and (23); an action of sitting down inherently includes motion from outward to inward.

In the sentences in (19), (22) and (23), expressions of change of physical position and spatial extent are allowed to co-occur with path phrases. At first glance, it appears that the change of physical position and spatial extent exhibits a distinct path, and anchored motion described by the path phrases represents another distinct path. The two paths, however, constitute a single unified path because the change of physical position and spatial extentingerently involves the anchored motion; the path phrases further specify the change of physical position and spatial extent.

In summary, the UPC is a constraint that bans a single clause from displaying more than one distinct path. When a change of state expression and a change of location expression co-occur in a single clause, one has to further specify the other; otherwise, each of them would represent a distinct path, violating the UPC. Anchored motion can further specify spatial extension. For this reason, no UPC violation arises when a sentence includes an expression that denotes spatial extension and a path phrase that describes anchored motion. (Note 5)

\subsection{The Under-Specification of Shape and the Loss of Physical Integrity}

In this subsection, I argue that anchored motion can be involved not only in the change of physical position and spatial extent but also in the change of state in terms of physical separation (i.e., a process in which an entity divides into different parts). Verbs that denote a change of physical position or spatial extent (such as spread) and those that denote physical separation (such as shatter) share the same semantic property. Both types of verbs are concerned with the meaning of physical shape or configuration. Furthermore, the exact shape or configuration is underspecified, and it can be specified by additional elements. Thus, the sentence in (25a) can be uttered in any context where the area occupied by the fire is now larger than before. Similarly, the sentence in (25b) is acceptable when the vase broke into pieces, regardless of whether its pieces are apart.

(25) a. The fire spread quickly.

b. John shattered the vase with a hammer.

The situations that can be described by these sentences are schematically exemplified below.

(26) a.

The fire spread quickly.
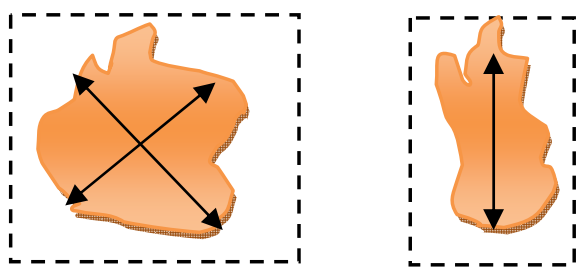

b. John shattered the vase with a hammer.
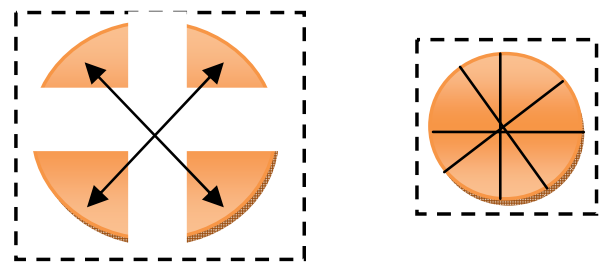
In the figures, the squares composed of broken lines express the configurations of space occupied by the fire or (pieces of) the vase. Arrows represent directions (i.e., anchored motion) of the fire spreading and the physical separation of the vase. In the left figure of (26b), for example, the centre of the figure constitutes an area that is anchored, and the extension represented by the arrows involves anchored motion. In this way, anchored motion does not necessarily include a part that is physically anchored at the original position.

Sentence (25a) may describe the situation in the left square of (26a), where the fire spread in all directions, as well as the situation depicted in the right square of (26a), where it spread vertically. Likewise, sentence (25b) may express the situation of the left square in (26b), where the vase broke into pieces and the pieces spread in all directions and that of the right square in (26b), where the vase broke into pieces although the pieces remain together.

Note that the size or configuration of the area occupied by the fire or (pieces of) the vase varies with the way the fire spread or the vase broke, respectively. How the fire spread or the vase broke is the underspecified part of meaning codified by the verb spread or shatter. This underspecified part of meaning can be further specified by expressions that are concerned with the change of spatial extent, such as out and all over the floor, as I will show in the following subsections. Crucially, the further specification using these expressions is possible because these verbs evoke the size or configuration of an object that is under-specified. For example, the size (or shape) of the fire that is spreading is not fixed and can be of various sizes depending on the context. Likewise, the configuration occupied by the vase that broke is not fixed, and the pieces may define various configurations depending on the context.

The under-specification of the size or configuration of an object follows from the loss of physical integrity of the object. The loss of physical integrity is the state in which the size or configuration defined by an object is not fixed due to the physical property of the object. An object that has lost physical integrity has the potential for a change in size or configuration. A shattered vase could keep its shape by chance, as shown in the right square in (26b), or it could lose shape and come apart, as shown in the left square in (26b). The same is true of fire. Fire that is spreading has the potential for change in size because it does not have a fixed shape.

In contrast, verbs that do not express the loss of physical integrity, such asbend, do not have the potential for a change in spatial extent. For this reason, they are incompatible with expressions that are relevant to spatial extension.

To summarisethis subsection, physical integrity is the key property that determines the compatibility of verbs with expressions that are concerned with spatial extension. Verbs that denote the loss of the physical integrity of an object have the potential for entailing anchored motion. Such verbs are exemplified by those denoting spatial extension (such as spread) and physical separation (such as shatter). Thus, verbs of spatial extension and those of physical separation are parallel in that they share the loss of physical integrity. The parallelism between the verbs of spatial extension and those of physical separation will be confirmed by two pieces of evidence, which are shown in sections 3.2.1 and 3.2.2.

\subsubsection{Compatibility with a Result Phrase Denoting Spatial Extension}

First, my proposal that change of state verbs such as break and shatter and verbs such as spread, which we will call spatial extension verbs hereafter, may share spatial extension meaning can be supported by the consistency of these verbs with the result phrase apart.

(27) a. Trent leaped to the portside bilge pump, desperate to clear the remaining water from the hull before its weight broke the vessel apart.

b. $\quad$ Another board shattered apart and fell inwards in shards.

(BNC, italics are mine)

The result phrase apart refers to a state of being separated by a distance. It has been observed that change of state verbs such as break and shatter can appear with result phrases that further specify the meaning entailed by the verbs (Levin \& Rappaport, 1995; Washio, 1997; Tortora, 1998, among others); the fact that these verbs are consistent with apart suggests that the meaning ofphysical separation is entailed by the verbs. The change of state verb bend, by contrast, does not include the meaning of the physical separation of an object, and apart is infelicitous as a result phrase:

* John bent the branch apart.

(In the interpretation where "John caused the branch to bend, as a result of this, it broke apart.")

In this way, change of state verbs that can co-occur with apart are restricted to those that entail physical separation. Spatial extension verbs are also compatible with the result phrase apart: 
(29) a. He spread his arms apart in a welcoming gesture,...

b. He stretched his arms apart to emphasize his point.
(G. Lamberson, Personal Demons)

(J. Cotton, Image of the Beast)

The verbs spread and stretch in these sentences denote spatial extension. In sentences (29a) and (29b), the referents of his arms move away from each other. The state of his arms being away from each other is further specified by the result phrase apart.

In this way, change of state verbs such asbreak and spatial extension verbs such asspread may co-occur with the result phrase apart, which further specifies the spatial extension meaning of the verbs. (Note 6)

In the following subsection, we observe the second piece of evidence for the parallelism in terms of spatial extension between these two types of verbs.

\subsubsection{Compatibility with Spatial Extension Expressions}

Expressions with out or all over (e.g., all over the floor), which we call spatial extension expressions hereafter, go along with verbs that display spatial extension, as shown below:

(30) a Spread out the newspaper to read.

b. He stretched out on the couch.

(Lindner, 1983:99)

(31) a. Once the lampshade fell from the ceiling and the glass broke and spread all over the floor.

(G. Lunsford, Georgia Is on My Mind)

b. We put new carpet in our family room about ten years ago and it has stretched all over the place.

(R. Rouse,Life Is...)

In sentences (30) and (31), the spatial extension expressions refer to spatial extension exhibited by the verbs spread and stretch. In (30), for example, out specifies a change of location that is involved in the spatial extension of the referents denoted by the newspaper and he. In (31), likewise, the motion that accompanies the spatial extension of the referents of the glass and new carpet is referred to by the spatial expressions all over the floor and all over the place, respectively. In this way, verbs that express spatial extension are compatible with spatial extension expressions that show the change of location inherent in the spatial extension.

In the same way as the result phrase apart, which we have discussed in section 3.2.1, these spatial extension expressions cannot appear with the verb bend, which does not include the meaning of spatial extension:

(32) a. * John bent the branch out on the floor.

(in the interpretation where "John broke the branch into pieces by bending it, as a result of this, the pieces spread out on the floor.")

b. * John bent the branch all over the floor.

(in the interpretation where "John broke the branch into pieces by bending it, as a result of this, the pieces spread all over the floor.")

Based on the contrast in acceptability between (30)-(31) and (32), let us assume that change of state verbs that are compatible with spatial extension expressions are restricted to those that entail spatial extension. If this assumption is correct, the following data verify that verbs of physical separation also include the meaning of spatial extension.

(33) a. The side mirror was crushed off and the driver's window broke out.

(D. Meyer, Life Is a Road, Ride It Hard!)

b. They think the windows shattered out because...

(R. Cragin, Wicked Winds)

(34) a. $\quad$... you might have seen an object break into pieces all over the floor, then slowly vanish.

(D. Franson,2D Artwork and 3D Modeling for Game Artists)

b. $\quad$ The china shattered all over the deck.

(D. Hinton, Letters from the Dead)

The verbs break and shatter in these sentences express the physical separation of an entity into more than one piece, and the spatial extension expressions display spatial extension. In the sentences in (33), the spatial extension expression out modifies the change of location involved in the spatial extension of the windows in terms of physical separation. Likewise, all over in the sentences in (34) exhibits motion that is included in the physical separation denoted by the verbs. 
In this way, verbs of physical separation (e.g.,break) and those of spatial extension (e.g.,spread) can co-occur with spatial extension expressions that may refer to spatial extension (e.g.,out and all over).

To summarise this section, verbs of physical separation and those of spatial extension are both compatible with result phrases and spatial extension expressions that can refer to spatial extension. (Note 7) This parallelism between these two types of verbs strongly suggests that verbs of physical separation may include the meaning of spatial extension. In section 3.1, we observed that verbs of spatial extension are compatible with path phrases because they inherently include motion, which is called anchored motion. Then, we can predict that verbs of physical separation may involve anchored motion, and they are compatible with path phrases when the path phrases refer to the anchored motion. In the next section, we argue that this prediction is correct.

\section{Separation as Spatial Extension}

As we have observed in section 1, many researchrs have supposed that the sentences in (14)-(16) are counterexamples to the UPC because they include two distinct changes in a single clause:

(35) a. The butcher sliced the salami onto the wax paper.

b. Joey grated the cheese onto a serving plate.

c. Sam shredded the papers into the garbage pail.

(36) a. The cook cracked the eggs into the glass.

b. $\quad$ Daphne shelled the peas onto the plate.

Kelly broke the branch off the tree.

At first sight, these sentences are structurally similar to the following in that they include a path phrase exhibiting motion of an object:

John threw the ball into the room.

A close scrutiny of these two types of sentences, however, reveals that the sentences in (35)-(37) are crucially different from (38) in the nature of motion. Sentence (38) displays translational motion, which can be confirmed by the unacceptability of the following sentence:

$$
\text { \# John has thrown the ball into the room, but he still holds the ball in his hand now. }
$$

This sentence suggests that the ball moves into the room, so it cannot stay in John's hand after he threw it.

What is relevant in sentences (35)-(37), on the other hand, is anchored motion, where only a part of an object moves along a path.

It is easier to demonstrate this point with the sentences in (36) and (37). In (36), for example, only the content of eggs or peas moves along a path denoted by the path phrases; their shell parts remain anchored at a fixed location, say, in one's hand. In fact, the following sentence cannot describe the situation where "John broke the egg, and then, both the liquid and shell fell into the bowl":

(40) \# John broke the egg into the bowl.

In sentence (37), likewise, what moves from the tree is only the branch; the tree remains anchored at a fixed location, say, at the ground. Thus, the sentences in (36) and (37) involve anchored motion, and hence they satisfy the UPC.

This observation also holds true for the sentences in (35), as illustrated by the following sentences:

(41) a. Grate or cut the cheese into the sauce, reserving a little to grate over the top of the dish.

(A. Sammy, Aunt Sammy's Radio Recipes, the underline is mine)

b. John has sliced the carrot into the bowl, but he kept the half of it for tomorrow, so he didn't slice the half part.

The underlined parts in the sentences, in which the interpretation where the whole part of the cheese or the carrot move along a path is cancelled, do not contradict the former part of the sentences. (Note 8) This fact explicitly shows that the sentences in (35) entail the motion of only part of an object; the whole object does not have to move along a path. The rest of the object can be considered anchored at a fixed location. The path phrases in (35), therefore, refer to anchored motion, and these sentences are in conformity with the UPC. 
When path phrases in sentences such as (41) refer to translational motion, these sentences become unacceptable, as illustrated by the following examples:

$$
\text { Mary cut the sausage into the pan. }
$$

a. (Acceptable in the interpretation where "Mary put a sausage into the pan while cutting it little by little.")

b. (Unacceptable in the interpretation where "Mary put cuts in a sausage (but the sausage is not cut into pieces) and put it in the pan.")

The sentence in (42) is acceptable in the interpretation shown in (42a) but not in the interpretation in (42b). The interpretation in (42a), in which only part of a sausage goes into the pan while Mary cuts the sausage, explicitly includes the anchored motion of the sausage. On the other hand, the interpretation in (42b), in which the whole sausage goes into the pan, involves the translational motion of the sausage. The unacceptability of sentence (42) in the interpretation of (42b) can be attributed to the translational motion of the sausage.

In this section, we have observed that the sentences in (35)-(37) include anchored motion, but not translational motion. This means that the path phrases in these sentences further specify the change of state denoted by the verbs. That is, the path phrases refer to motion inherent in the processes of spatial extension in terms of physical separation. As a result, these sentences include only one path, and no UPC violation arises.

The spatial extension expressed by the sentences in (35)-(37) can be represented in the following figures:

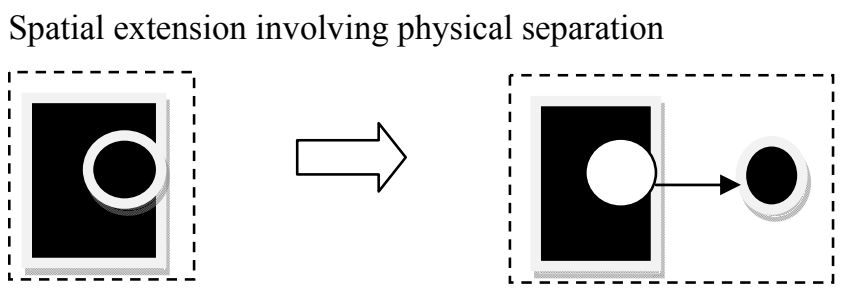

In these figures, the thick white arrow displays a transition of situations. The thin arrow represents anchored motion of the circle. In the left square, the black square and the circle constitute a unit. The right square exhibits spatial separation of the circle from the black square. The dotted squares denote the ranges of space covered by the black square and the circle. The figures in (43) show that the black square and the circle in the separated state occupy a larger area than in the unified state.

\section{Further Consideration: Melt}

In the previous section, we observed that the sentences in (35)-(37), which are apparently problematic for the UPC, are not true counterexamples to it. The acceptability of these sentences can be explained by saying that the path phrases in these sentences refer to anchored motion.

Verbs that are compatible with path phrases, however, are not restricted to those that denote physical separation. In fact, it has been noted that verbs that denote a change of state in terms of melting also go along with path phrases:

The chocolate melted out of the box.

(Jackendoff, 1990:241)

In this sentence, the change of state verb melt co-occurs with a path phrase, and this sentence appears to violate the UPC.

A close investigation of this example, however, unveils that the path phrase in this sentence refers to anchored motion, and hence this sentence does not violate the UPC. As shown in the following sentence, the verb melt is compatible with the result phrase apart and with the spatial extension expressions out and all over the grate, all of which display spatial extension:

John melted the handle and the cup apart.

(In the interpretation where "John caused the handle and the cup (which are made of ice) to become apart by melting it.")

John melted the ice out on the floor.

(In the interpretation where "John melted the ice, and then the water spread out on the floor.") 
In just minute the plastic was melted all over the grate.

(J. Holmes, There Should Have Benn Roses, italics are mine)

Given the consistency of the verb melt with apart, out and all over the grate, we can predict that the sentence in (44) includes anchored motion. This prediction is borne out by the following sentence, which shows that sentence (44) does not entail translational motion of the chocolate:

The chocolate has melted out of the box, but there is still some chocolate in it now.

The underlined part in sentence (48) does not contradict the former part of the sentence. This fact explicitly shows that the motion included in (44) is not translational motion but anchored motion.

Note that the verb freeze, which is opposite in meaning to the verb melt, cannot co-occur with the path phrase out of the bottle in the following sentence:

(51) * The water froze out of the bottle. (in the sense "The water got out of the bottle by freezing")

(Jackendoff, 1990:241)

The unacceptability of this sentence follows because the verb freeze does not entail the loss of physical integrity and cannot describe spatial extension, which inherently involves anchored motion. As a result, the path phrase cannot further specify the meaning of the verb, and the unacceptability of (51) results.

\section{Conclusion}

In this paper, I have argued that the UPC effect comes from the nature of event structure: a single clause may not include more than one distinct result. On the basis of the theory of event structure provided by Rappaport \& Levin (1998), I have argued that the sentences in (35)-(37), which are considered counterexamples to the UPC by many researchers, are only apparent counterexamples. Although these sentences include a change of state verb and a path phrase in a single clause, and they appear to contain two distinct paths, I have shown that the change of state exhibited by the verb and the motion denoted by the path phrase constitute a single unified path. Specifically, I have shown that the path phrases in these sentences refer to a motion that is inherently involved in the change of state denoted by the verbs. As a result, path phrases are allowed to co-occur with change of state verbs in these sentences, without violating the UPC. The analysis presented in this paper thus provides empirical evidence for the event-based analysis of the UPC effect, suggesting that human language is devised as sensitive to the mechanism of event structure.

\section{Acknowledgements}

I would like to express my thanks to the following people for their invaluable comments on earlier versions of this paper: NobuhiroKaga, Yukio Hirose, Masaharu Shimada, Naoaki Wada, Hiromi Onozuka.Thanks are also due to SoulefBatnini and Jess Timms for kindly acting as informants.Needless to say, any remaining errors and shortcomings are mine.This work is supported by JSPS Research Fellowship for Young Scientists (JSPS Research Fellow DC2) and by a Grant-in-Aid for JSPS Fellows.

\section{References}

British National Corpus [BNC].

Collins COBUILD Advanced Learner's English Dictionary (5th edition) [COBUILD], London: HarperCollins Publishers.

Goldberg, A. (1991). It can't go down the chimney up: Path and the English resultative. BLS 17, 368-378.

Goldberg, A. (1995). Constructions: A Construction Grammar Approach to Argument Structure. Chicago: The University of Chicago Press.

Goldberg, A. (2001). Patient arguments of causative verbs can be omitted: The role of information structure in argument distribution.Language Sciences 23, 503-524. http://dx.doi.org/10.1016/S0388-0001(00)00034-6

Jackendoff, R. (1990). Semantic Structures. Cambridge, Mass: MIT Press.

Levin, B. \& M. Rappaport Hovav. (1995). Unaccusativity:At the Syntax-Lexical Semantics Interface. Cambridge, Mass: MIT Press.

Lindner, S. (1982). What goes up doesn't necessarily come down: The ins and outs of opposites.CLS 18, 305-323.

Lindner, S. (1983). A Lexico-Semantic Analysis of Verb-Particle Construction with Up and Out. Indiana: Indiana University Linguistics Club. 
Matsumoto, Y. (1996a). Subjective-change expressions in Japanese and their cognitive and linguistic bases. In E. Sweetser\& G. Fauconnier (Eds.),Spaces, Worlds, and Grammar(pp. 124-156), Chicago:The University of Chicago Press.

Matsumoto, Y. (1996b). Subjective motion and English and Japanese verbs.Cognitive Linguistics 7(2), 138-226. http://dx.doi.org/10.1515/cogl.1996.7.2.183

Matsumoto, Y. (2002). Siekiidookoobunniokeruimitekiseiyaku [Semantic constraints on the caused motion construction]. In Y. Nishimura (Ed.),JishooKoozoo [Event Structure] (pp. 187-211),Tokyo: University of Tokyo Press.

Matsumoto, Y. (2006). Constraints on the co-occurrence of spatial and non-spatial paths in English: A closer look at the Unique Path Constraint. Paper presented atFourth International Conference on Construction Grammar, University of Tokyo.

Okuno, K. (2003). The patient constraint on the resultative construction and the construction fusion.Studies in the Humanities, Faculty of Humanities, Hirosaki University, 158-170.

Pi, C. T. (1999). Mereology in Event Semantics, Ph.D. Dissertation, McGill University.

Pinker, S. (1989). Learnability and cognition: The acquisition of argument structure. Cambridge, Mass: MIT Press.

Pustejovsky, J. (1991). The syntax of event structure. Cognition 41, 47-81. http://dx.doi.org/10.1016/0010-0277(91)90032-Y

Ramchand, G. (2008). Verb meaning and the lexicon: A first phase syntax, Cambridge, Mass: Cambridge University Press. http://dx.doi.org/10.1017/CBO9780511486319

Randall, J. (2010). Linking: The geometry of argument structure. New York: Springer. http://dx.doi.org/10.1007/978-1-4020-8308-2

RappaportHovav, M. \& B. Levin. (1998). Building verb meanings.In M. Butt \& W. Geuder (Eds.), The Projection of Arguments: Lexical and Compositional Factors(pp. 97-134), Stanford: CSLI Publications.

RappaportHovav, M. \& B. Levin. (2001). An event structure account of English resultatives.Language 77, 766-797. http://dx.doi.org/10.1353/lan.2001.0221

Ritter, E. \& S. Rosen. (1998). Delimiting events in syntax.In M. Butt \& W. Geuder (Eds.), The Projection of Arguments: Lexical and Compositional Factors(pp. 135-164), Stanford: CSLI Publications.

Simpson, J. (1983). Resultatives.In L. Levin, M. Rappaport Hovav\& A. Zaenen (Eds.), Papers in Lexical-Functional Grammar(pp. 143-158), Indiana: Indiana University Linguistics Club.

Talmy, L. (2000). Toward a Cognitive Semantics Vol. II, Cambridge, Mass: MIT Press.

Tortora, C. M. (1998). Verbs of inherently directed motion are compatible with resultative phrases.Linguistic Inquiry 29, 338-345. http://dx.doi.org/10.1162/ling.1998.29.2.338

Vendler, Z. (1957). Verbs and times. The Philosophical Review 66(2), 143-160. http://dx.doi.org/10.2307/2182371

Washio, R. (1997) .Resultatives, compositionality and language variation. Journal of East Asian Linguistics 6, 1-49. http://dx.doi.org/10.1023/A:1008257704110

Yasuhara, M. (2012). A closer look at the Unique Path Constraint from the point of view of spatial extension.Tsukuba English Studies 31, 41-63.

Yasuhara, M. (2013). One eventuality per subevent: An event-based account of argument structure.Language Sciences 40, 251-262. http://dx.doi.org/10.1016/j.langsci.2013.06.004 


\section{Notes}

Note 1. Tortora (1998) argues that the verb break can co-occur with result phrases that further specify the meaning of the verb. Observe the following data:

(i) a. John broke the vase into pieces.

b. * Bill broke the vase worthless.

(Jackendoff, 1990:240)

The meaning of the verb break inherently includes physical separation but not a change in value; the former is exhibited by the result phrase into pieces and the latter by the result phrase worthless. The verb break entails physical separation of an object, and the result phrase into pieces further specifies this meaning. The incompatibility of the verb break with worthless can be explained by saying that the former does not entail the meaning of the latter; the former cannot be further specified by the latter.

Note 2. The sentences in (ia) and (iia) can be paraphrased with (ib) and (iib), respectively.

(i) a. Sam kicked Bill black and blue.

b. Sam made Bill black and blue by kicking him.

(ii) a. Sam kicked Bill out of the room.

b. Sam caused Bill to go out of the room by kicking him.

These paraphrases show that the change of state (Bill's becoming black and blue) and the change of location (Bill's going out of the room) are both caused by Sam's kicking. That is, Sam's kicking is a causing event, and Bill's change of state and his change of location are result events.

Note 3. This definition is based on Goldberg's (1991:373) statement:

[T] he object undergoing the change remains anchored at a fixed location, while rearranging parts of its extension in space.

This statement is a part of the explanation of the sentences in (19).

Note 4. As space does not permit a full investigation of grammatical phenomena that involve spatial reduction, I focus for the remainder of this paper on grammatical phenomena that are concerned with spatial extention.

Note 5.Generally, multiple modifiers that further specify the time or place may co-occur in a single clause:

(i) a. John arrived [in 2001] [in March] [during the third week] [in the afternoon] [at 2:00 pm].

b. $\quad$ The train arrived [in NYC] [at Penn Station] [on track 31].

The modifiers in brackets specify the time of John's arrival in (ia) and the place where the train arrived in (ib). This also holds true of modifiers that display a change of state or location in terms of spatial extension:

Pity him, be reverent to the clay that can no more resist your touch, cover him deep from all your senses, and stand up straight into the sun again with your head beyond the high clouds.

(The English Review Vol. 33, underline is mine)

The underlined part in this example includes two path phrases up and into the sun and the result phrase straight. The result phrase refers to the change of position, and the two path phrases specify the direction of standing. All these modifiers further specify the meaning of the verb stand.

Note 6.Change of state verbs such asbreak and change of spatial extent verbs such asspread are also compatible with result phrases such as into pieces, which specify the state of being separated in space:

Maggie \{broke / split / cracked / splintered / crumbled / divided / shattered $\}$ the bowl into a thousand pieces.

(Jackendoff, 1990:117)

(ii) a. The jute is spread in pieces,...

(P. Sharp, Flax, Tow, and Jute Spinning)

b. The battleship became enlarged, spreading into pieces then exploded from the inside out.

(L. Cabral, Oisin)

Result phrases such asinto pieces denote a state of being spatially separated, and the sentences in (i) explicitly show that verbs such as break entail physical separation. The physical separation denoted by result phrases such asinto pieces is also consistent with change of spatial extent verbs, as shown in (ii). The fact that verbs of change of state in terms of physical separation and those that refer to spatial extension are both compatible with result phrases such 
asinto pieces supports my proposal that these two types of verbs may share spatial extension meaning.

Note 7. The parallelism between verbs of physical separation and those of spatial extension can also be verified by their compatibility with subjective motion expressions. Subjective motion expressions denote the spatial range of an entity such as a road, and they can be considered related to spatial extent (cf. Jackendoff (1990)). For this reason, this type of expression is compatible with change of spatial extent verbs, as shown below:

(i) a. The road extends from San Francisco to Los Angeles.

(Matsumoto, 1996a:141)

b. The road widens at the junction.

(Matsumoto, 1996b:185)

c. Down below, beyond wisps of vapor steaming past, ocean spreads out to the horizon.

(W. Campbell, Apotheosis)

Sentence (ia), for example, expresses that the road ranges from San Francisco to Los Angeles.

Some verbs of physical separation are also compatible with subjective motion expressions. The following sentences include the change of state verbs split and break:

(ii) a. Go right onto North Bloomfield Road and follow it up to the top of the hill where the road splits. (L. Austin, Mountain Bike! Northern California)

b. From Copper Basin, several other roads break out to the south, east, and north.

(T. Lopez, Idaho, a Climbing Guide)

Sentence (iia) indicates that the road branches at the top of the hill. Sentence (iib), likewise, expresses that several roads run in several directions from Copper Basin.

In this way, both verbs of physical separation and verbs of spatial extension are compatible with subjective motion expressions. The data in (i) and (ii) further support the parallelism between the two types of verbs in terms of spatial extension.

Note 8. Similar observations are made by Rappaport \& Levin (2001) and Pi (1999). Regarding sentence (i), Pi (1999:191), for example, gives the following remarks in (ii):

Greg cut the carrot into the bowl.

Note as well that the process may be one stretch of the cutting action, but the individual pieces fall independently and successively into the bowl. Thus, each instance of a piece falling into the bowl might be considered to be a minimal part to the process of to cut a carrot into the bowl.

In the quotation in (ii), Pi observes that the event of cutting the carrot into the bowl includes several actions of cutting it. The pieces of it made by each cutting action fall into the bowl successively. Because the path phrase into the bowl in (i) refers to the motion of a part of the carrot, the motion can be considered anchored motion. 